\title{
Key Technology Research of Computer-Aided Three-Dimensional Modeling for the Bridge
}

\author{
Ying Wang ${ }^{1, a}$, Jianhua Guo ${ }^{1, b}$ \\ ${ }^{1}$ School of Tianjin University, Tianjin 300072, China; \\ asliverjane@163.com, bguojianhua_tx@126.com
}

Keywords: Bridge; Autodesk CAD; Three-Dimensional Modeling; Computer-Aided Design;

\begin{abstract}
With the rapid development of science and technology, three-dimensional modeling technology bridges will continue to progress, which is widely used in bridge engineering, bridge design can contribute to enhance the quality, thus contributing to the sustainable development of the bridge project. Three-dimensional computer model of the structure of the bridge as the implementation of key aspects of computer-aided design, and its significance in the integration of computer-aided design system is particularly prominent. For the bridge structure, since the structure of complex, low degree of standardization, three-dimensional modeling of real structures is very difficult. Based on Autodesk CAD actively to explore three-dimensional design work and the application of the bridge, the whole system is sufficient to demonstrate the effectiveness and feasibility of three-dimensional modeling of the bridge.
\end{abstract}

\section{Introduction}

Three-dimensional computer model of the structure is the key to achieve computer-aided design, and its significance in the integration of computer-aided design system is particularly prominent. For the bridge structure, since the structure of complex, low degree of standardization, three-dimensional modeling of real structures is very difficult. Meanwhile, the computer modeling technology is relatively backward, many CAD systems have to mechanically follow the model of two-dimensional design engineers have been accustomed to and the corresponding expression methods [1-2]. Due to the lack of the necessary three-dimensional model of the real structure, limiting the function of computer-aided design system and the potential for further development. Thus, with the progress of computer technology, three-dimensional modeling techniques and methods to explore the bridge structure has a certain significance. It not only improves the ease of use of the system from a visual point of view, more from the perspective of the data model to further improve the functionality of the system.

Thus, three-dimensional design technology is the development trend of the current computer-aided design technology, machinery, construction, chemical, petroleum and other industries has been relatively successful design application, and become an important tool in market competition. Oriented architecture lifecycle integrated modeling technology in the construction industry has been widely recognized, Autodesk CAD based actively explore three-dimensional design work and the application of the bridge.

\section{Computer-aided three-dimensional modeling techniques}

The establishment of three-dimensional model space objects, in essence, is how to define and express a three-dimensional object within a computer. CAD / CAM field of study on this issue started earlier and developed more mature group of three-dimensional geometric representation of an object, such as a surface representation, spatial segmentation method and structure representation method. Based on three-dimensional modeling system of these models, such as AutoCAD, 3DS, Micro-Station, etc., it has been widely used [3]. However, due to CAD data management system itself is limited, it is difficult to effectively manage large quantities of data and three-dimensional space to achieve their operating and analysis, most of the purely visual model. 
Geometry is the most intuitive sense engineer, geometric modeling is good or bad is a measure of an important indicator of the whole bridge construction. Because of the complexity of the bridge structure of the model, the entire model definition process is divided into three steps, as shown in FIG.

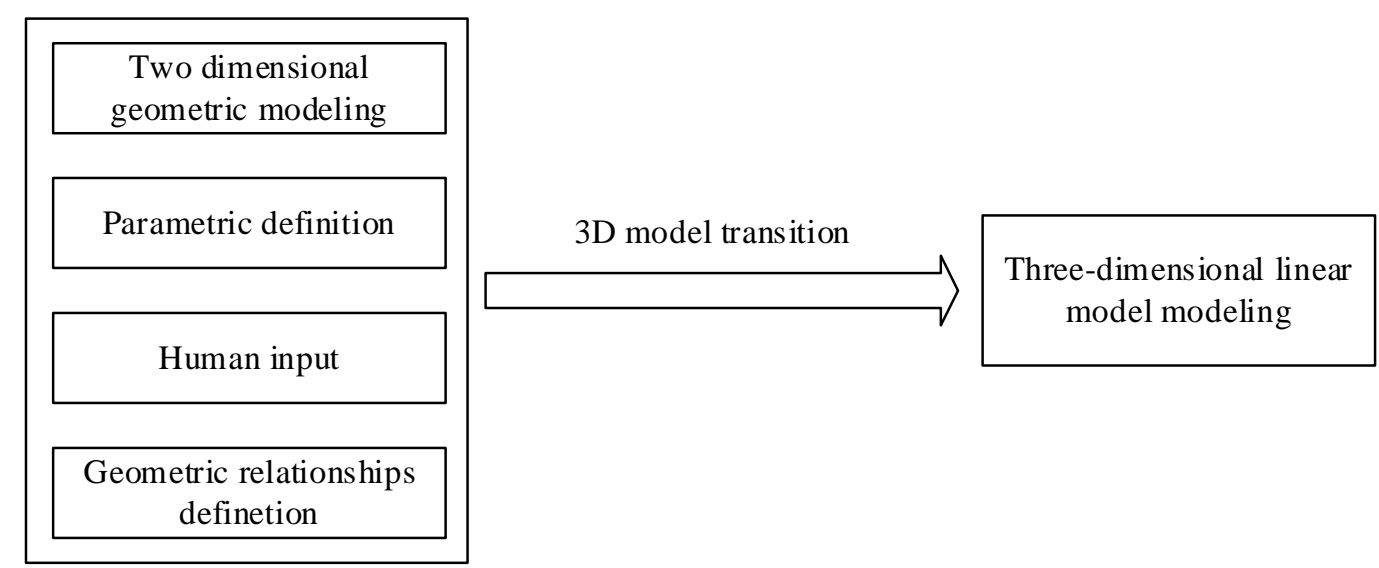

Figure 1 Computer-aided three-dimensional modeling technological processes

Three-dimensional CAD data model based on the bridge also has two meanings: First, the traditional boundary model to represent the overpass surface shape and topology using point line side has been one of the bridge structure represents a space object that will unify all polygonal surfaces, each polygon edges are straight line segments. For the surface, it is divided into smaller polygons, so that the data structure is greatly simplified, and the surface here was established by the WYSIWYG topology on digital imaging and implemented depends feature data sources include directly acquired feature point information and constraint information characteristic surface. Feature information that is geometry data of each characteristic point boundary bridge, constraint information characteristic surface is the surface topology information, namely one surface which is composed by the space segment, and then extended to the spatial topology information plane by an ordered set of three-dimensional feature point to describe. In addition, the modeling of such complex bridge structures also need the help of the bridge route CAD model (or design data), that information can not be obtained from the vector image to bridge some of the surface, you can restore the method according to the design data vector data complement its vector information. CAD model of thinking to build bridges in the bridge sections into one, and then a combination of these sections into one surface]. The difference is that here the surface is to establish a strict topological relationships end to end, and CAD each faces are isolated from each other have cracks. Data from the above two parts can create vector data model of the bridge, then the bridge vector data model and the resulting digital terrain model and image texture information overlay, get a real three-dimensional model of the bridge.

\section{Bridge dimensional geometric model design based Autodesk CAD}

Autodesk CAD technology in the field of civil engineering is currently a commonly used mapping tools, references Autodesk CAD technology in the process of three-dimensional modeling of the bridge, forming the intersection of Autodesk CAD interface enables the establishment and read DXF files. DXF interface is mainly used to build a cross-section of the bridge draw, for some special structure and built-section can be achieved by Autodesk CAD, Autodesk CAD by the cross-sectional designed by computer, and then imported into three-dimensional modeling system through the interface, note that the graphical properties are mostly imported from DXF linear object, so it needs to be converted after processing, it can be successfully used in the three-dimensional modeling system [4].

The main objective of this process is described in the state of the entire two-dimensional geometry of the bridge, arranged some geometry-related information, such as control points, support and resistance and load loading points and other information, in order to define the model geometry definition, the system at the same time use template parameter definitions and human 
interaction are two input methods, including the entire facade of the bridge model, the cross section and plane definitions [5]. As for the geometry defined two-dimensional model, using the method of geometry modeling techniques, the main advantage of the construction method of object-oriented graphics system complex graphics objects based on two-dimensional geometric modeling Autodesk CAD is shown in Figure 2.

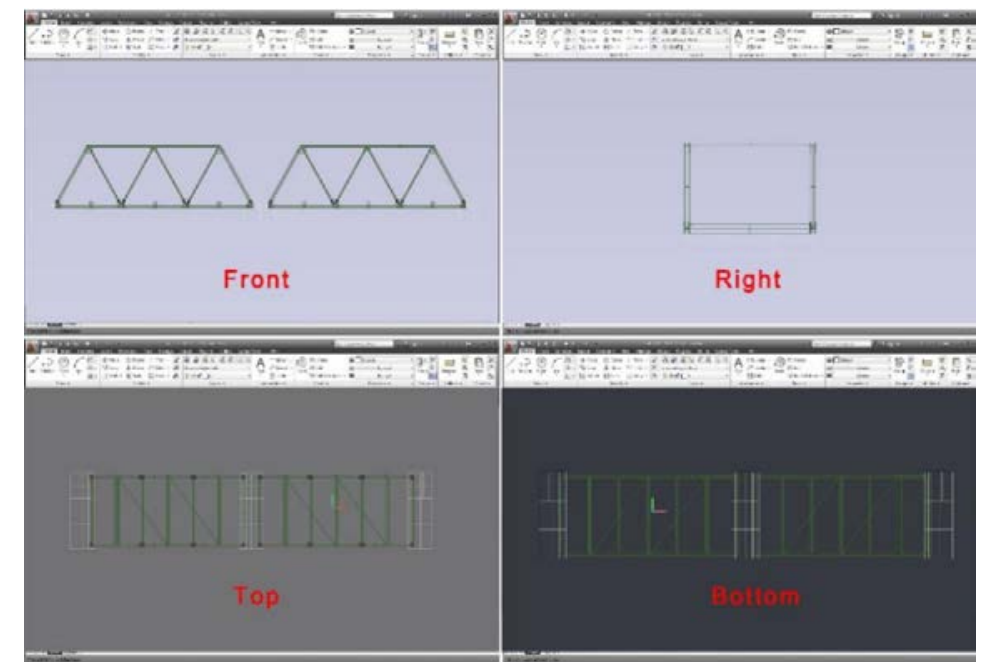

Figure 2. Based on the two-dimensional geometric modeling of Autodesk CAD

\section{Bridge three-dimensional modeling application based on Autodesk CAD}

In computer-aided three-dimensional modeling, two-dimensional model is the basis of information generated three-dimensional model, and for the purposes of computer-aided design system, a truly effective geometrical model requires three-dimensional model information. It is necessary to establish a model converter between two and three dimensions, and this model converter is based on a two-dimensional model and solid geometric relationship above. Two-dimensional state entity data includes mutual relations and their own characteristics, all of which can be converted to the corresponding three-dimensional and three-dimensional graphics features of the relationship between three-dimensional graphics converter main principle in the use of computer graphics technology to achieve projection transformation.

Wireframe model is the basis of three-dimensional geometric analysis model, three-dimensional graphics tool through a three-dimensional graphics operations to control, the graphics operations provides a basic graphics operation functions, such as adding an entity, delete and modify the three-dimensional entity, select the entity and zoom and other functions. Autodesk CAD-based three-dimensional modeling of the bridge is shown in Figure 3. 


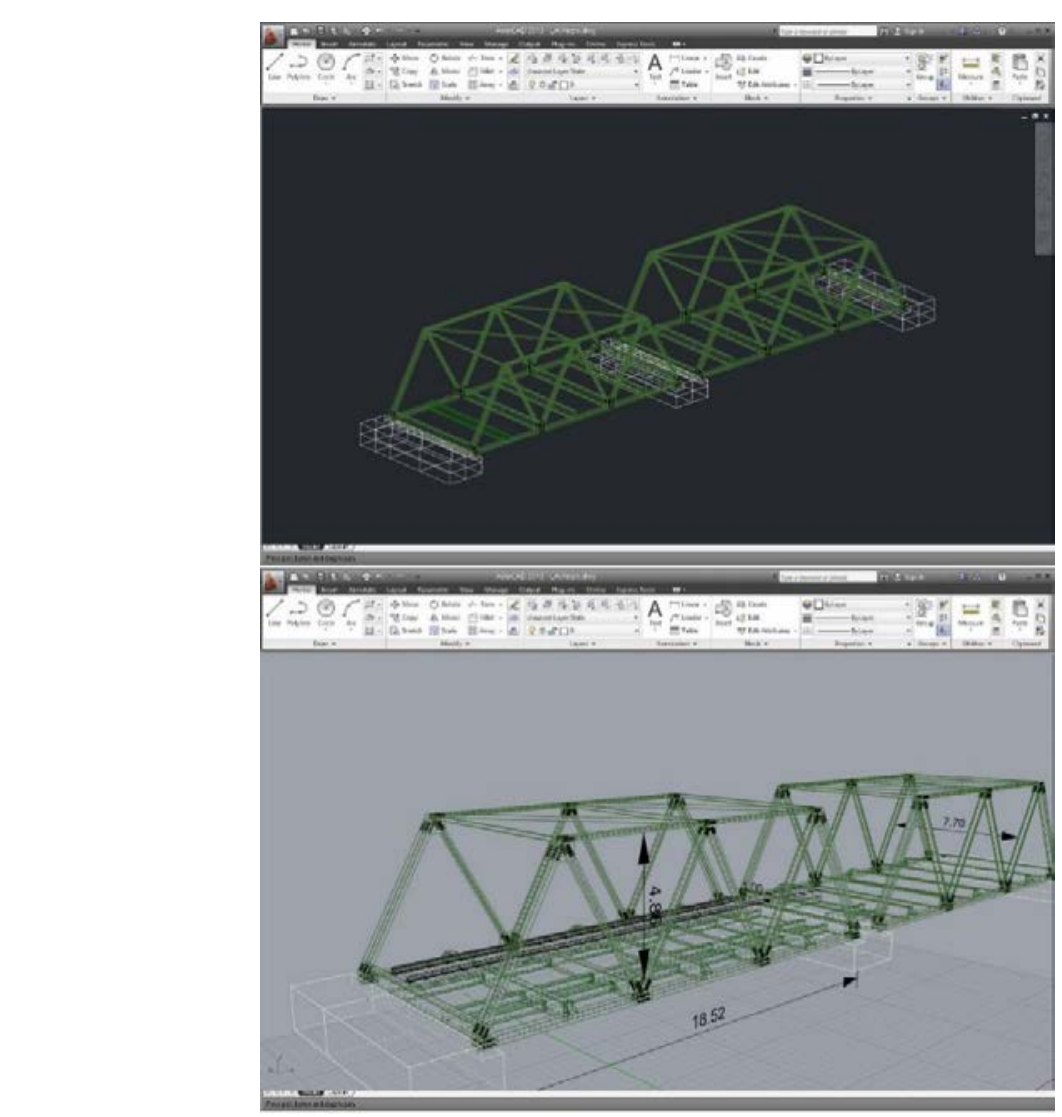

Figure 3. Autodesk CAD-based three-dimensional modeling application bridge

\section{Conclusion}

Computer-aided three-dimensional modeling technology is a key technology bridge construction. In part, characterized by parametric design greatly simplifies the structure of geometric modeling process, and is very easy for users to understand and use. Different models for bridge construction object-oriented database management, and analyze information obtained by each construction stage key index words, in this way to simulate the entire construction process has strong support and flexibility. Based on Autodesk CAD actively explore three-dimensional design work and the application of the bridge, the whole system is sufficient to demonstrate the effectiveness and feasibility of three-dimensional modeling of the bridge.

\section{References:}

[1] Chung W, Sotelino E D. Three-dimensional finite element modeling of composite girder bridges[J]. Engineering Structures, 2006, 28(1): 63-71.

[2] Garcia M. Sedimentation engineering: processes, measurements, modeling, and practice[C]. ASCE, 2008.

[3] Martinez R V, Branch J L, Fish C R, et al. Robotic tentacles with three - dimensional mobility based on flexible elastomers[J]. Advanced Materials, 2013, 25(2): 205-212.

[4] Lee T L, Jeng D S, Zhang G H, et al. Neural network modeling for estimation of scour depth around bridge piers[J]. Journal of Hydrodynamics, Ser. B, 2007, 19(3): 378-386.

[5] Ren W X, Peng X L. Baseline finite element modeling of a large span cable-stayed bridge through field ambient vibration tests[J]. Computers \& structures, 2005, 83(8): 536-550. 